\title{
Rivaroxaban: Expanded Role in Cardiovascular Disease Management-A Literature Review
}

\author{
Muhammad Ajmal (iD, Jacob Friedman (D), Qurat Ul Ain Riaz Sipra $(\mathbb{D}$, and Tom Lassar \\ University of Arizona, College of Medicine, Tucson, AZ, USA \\ Correspondence should be addressed to Muhammad Ajmal; drajmal207@gmail.com
}

Received 1 September 2020; Revised 22 December 2020; Accepted 4 January 2021; Published 8 January 2021

Academic Editor: Victor Garcia

Copyright (c) 2021 Muhammad Ajmal et al. This is an open access article distributed under the Creative Commons Attribution License, which permits unrestricted use, distribution, and reproduction in any medium, provided the original work is properly cited.

\begin{abstract}
Direct oral anticoagulants (DOACs) are widely used for the prevention of stroke in nonvalvular atrial fibrillation, treatment of deep venous thrombosis and pulmonary embolism, and as prophylaxis after hip and knee surgery after approval by the Food and Drug Administration. In the last decade, DOACs were studied for various indications; this review is focused on rivaroxaban, a factor Xa inhibitor, which is used in an expanded evidence-based fashion for coronary artery disease, peripheral artery disease, heart failure, malignancy, and prophylaxis of deep venous thrombosis in acute medical illnesses.
\end{abstract}

\section{Introduction}

Vitamin K antagonists (VKAs) have been used for anticoagulation in humans since 1954 and were even prescribed to then sitting President Dwight Eisenhower after his myocardial infarction. However, due to various limitations of VKA (e.g., drug-drug interactions, narrow therapeutic window, and need for a blood test to monitor therapeutic effect), DOACs which affect specific targets factor Xa and/or factor II have emerged as a potentially preferred therapeutic strategy to overcome these limitations [1]. Rivaroxaban is a direct factor Xa inhibitor and has been studied in various thromboembolic and atherothrombotic conditions [1]. Factor Xa plays a key role in both intrinsic and extrinsic coagulation pathways leading to the downstream activation of thrombin and clot formation [2]. Rivaroxaban is a small molecule that reversibly inhibits both free and clot-bound factor Xa [2]. It has been used until recently mostly for nonvalvular atrial fibrillation (NVAF) and deep venous thrombosis or venous embolism, but its use is increasing in a growing variety of vascular conditions, notably coronary artery disease, peripheral artery disease, and thromboprophylaxis. The purpose of this review is to provide a comprehensive overview of rivaroxaban use for these rapidly broadening indications.

\section{Anticoagulation in Nonvalvular Atrial Fibrillation}

Atrial fibrillation is one of the most common clinically manifested arrhythmias, causing significant morbidity and mortality due to systemic arterial thromboembolism, particularly stroke [3]. Traditionally, warfarin has been used for anticoagulation in patients with NVAF who require anticoagulation based on the CHA2DS2 or CHA2DS2-VASc score [4]. Because of warfarin's narrow therapeutic window and the need for frequent therapeutic monitoring with the international normalized ratio (INR), DOACs have emerged as a promising anticoagulation strategy for patients with NVAF requiring long-term anticoagulation [5]. Rivaroxaban in the management of NVAF was initially studied in the ROCKET $\mathrm{AF}$ trial, which compared rivaroxaban to warfarin for cerebrovascular event prophylaxis and safety. In this trial, $20 \mathrm{mg}$ rivaroxaban daily for patients with creatinine clearance $(\mathrm{CrCl}) \geq 50 \mathrm{~mL} / \mathrm{min}$ or $15 \mathrm{mg}$ rivaroxaban daily in those with $\mathrm{CrCl}$ between 30 and $49 \mathrm{~mL} / \mathrm{min}$ was found to be noninferior to warfarin therapy in reducing thromboembolic events, with a significant reduction in intracranial hemorrhage and fatal bleeding [6]. The results of this trial led to the Food and Drug Administration's (FDA) approval of 
rivaroxaban in nonvalvular atrial fibrillation on November 4, 2011 [7]. The approval of rivaroxaban various indications in the USA and Europe is shown in Table 1.

The real-world experience based on postmarketing surveillance registry data also showed the safety and efficacy of rivaroxaban in atrial fibrillation patients with comorbidities of diabetes mellitus, chronic kidney disease, acute coronary syndrome, and cancer [8]. Like the ROCKET AF trial, rivaroxaban imparted a significantly lower risk of intracranial hemorrhage in systematic review and metanalysis (rivaroxaban $\mathrm{HR}, 0.64 ; 95 \% \mathrm{CI}, 0.47-0.86$ ) compared to warfarin therapy, while providing similar protection against stroke and heart attack [9]. In the systematic review and network meta-analysis comparing DOACs to warfarin, DOACs appear to be at least equivalent to warfarin in preventing stroke in atrial fibrillation with reduced risk of bleeding [10].

In the current American College of Cardiology (ACC)/American Heart Association (AHA)/Heart Rhythm Society (HRS) 2019 focused update on 2014 guidelines, rivaroxaban has a class 1 recommendation, with the level of evidence $\mathrm{B}$ in nonvalvular atrial fibrillation with a CHA2DS2-VASc score of 2 or higher in men and three or greater in women [11]. In the 2014 ACC/AHA/HRS guidelines, rivaroxaban, and other DOACs, had a prior class 1 recommendation with the level of evidence $B$ in nonvalvular atrial fibrillation and a CHA2DS2-VASc score of 2 or more in both males and females [12].

\section{Treatment of Deep Venous Thrombosis and Pulmonary Embolism}

Deep venous thrombosis (DVT) is a part of the spectrum which includes superficial thrombophlebitis and pulmonary embolism [13]. After heart attack and stroke, pulmonary embolism (PE) is the third most common cause of death worldwide [14]. Rivaroxaban was initially studied in two dose-finding studies, where a single daily dosage of rivaroxaban was found to be feasible as a treatment for DVT compared to low molecular weight heparin and VKA $[15,16]$. These studies led to the EINSTEIN program, consisting of three randomized trials of rivaroxaban. The first trial was for the treatment of acute DVT, the second for the treatment of acute PE, and the third for the continued treatment for those who received treatment for the acute DVT/PE and was continued with rivaroxaban for longer term $[17,18]$. The treatment protocol in the acute DVT and acute PE study was rivaroxaban $15 \mathrm{mg}$ twice daily for three weeks followed by $20 \mathrm{mg}$ daily. It was compared to low molecular weight heparin for the median of 8 days until warfarin was therapeutic with an INR level of $\geq 2$. In the continued treatment group, rivaroxaban was compared to the placebo with a dosage of $20 \mathrm{mg}$ daily. In the trials, it was concluded that rivaroxaban might be considered a safe and effective single drug for the treatment of venous thrombosis and venous thromboembolism $[16,17]$. This led to the approval of rivaroxaban by the FDA in 2012 [19]. Based on this data of rivaroxaban and the data on other DOACs, the American College of Chest Physicians, in their 10th version of guidelines, recommended to prefer treatment of acute DVT/PE with DOACs over VKAs.

Later, the EINSTEIN CHOICE Investigators studied the $20 \mathrm{mg}$ or $10 \mathrm{mg}$ of rivaroxaban daily and compared it to aspirin $100 \mathrm{~m}$ mg daily for extended treatment of venous thromboembolism after 6 or 12 months of initial treatment decided by the treating physicians. In this study, $20 \mathrm{mg}$ or $10 \mathrm{mg}$ daily rivaroxaban was associated with a significantly reduced risk of recurrent venous thromboembolism without an increased risk of bleeding when compared to aspirin [20]. The low dose of rivaroxaban, which is essentially the prophylactic dose, may be considered for longer-term treatment in patients who have already been treated with a full dose to prevent recurrent venous thromboembolism.

Early discharge within 24 hours after low-risk PE was studied in the HoT-PE (The Home Treatment of Patients with Low-Risk Pulmonary Embolism with the Oral Factor Xa Inhibitor Rivaroxaban) trial. Early discharge with rivaroxaban $15 \mathrm{mg}$ twice daily for three weeks followed by $20 \mathrm{mg}$ daily was found to be effective and safe in low-risk acute PE patients [21]. MERCURY PE (Multicenter Trial of Rivaroxaban for Early Discharge of Pulmonary Embolism from the Emergency Department), a randomized, multicenter trial, concluded that early emergency department discharge of low-risk PE patients on rivaroxaban results in significantly lower costs and shorter duration of initial and subsequent hospitalizations without an increase in serious adverse events [22].

The systematic review and network meta-analysis comparing the direct oral anticoagulants found that all DOACs are effective in reducing the risk of venous thromboembolism and preventing death related to venous thromboembolism, although the bleeding profile of apixaban was better compared to other DOACs [23].

\section{Postoperative Venous Thromboembolism Prophylaxis after Hip and Knee Surgery}

Venous thromboembolism (VTE) is a deadly complication after major orthopedic surgeries of hip and knee arthroplasty, and anticoagulants are used to prevent these complications [24]. To prevent VTE, low molecular weight heparin for ten days was used as a standard of care after hip and knee arthroplasty based on the recommendations of the Seventh American College of Chest Physicians recommendations on antithrombotic therapy [25]. This led to additional trials to find oral treatment to prevent VTE complications and associated morbidity and mortality with VTE. Four phase III randomized clinical trials, RECORD 14 (Regulation of Coagulation in Orthopedic Surgery to Prevent Deep Venous Thrombosis and Pulmonary Embolism), were conducted to evaluate rivaroxaban compared to enoxaparin. RECORD 1 compared rivaroxaban $10 \mathrm{mg}$ to enoxaparin $40 \mathrm{mg}$ daily for 35 days after hip arthroplasty. RECORD 2 compared rivaroxaban $10 \mathrm{mg}$ daily for 31-39 days to enoxaparin $40 \mathrm{mg}$ for 10-14 days after hip arthroplasty. RECORD 3 compared rivaroxaban $10 \mathrm{mg}$ daily to enoxaparin $40 \mathrm{mg}$ for 10-14 days after knee arthroplasty. Finally, RECORD 4 compared rivaroxaban $10 \mathrm{mg}$ daily for 
TABLE 1: Indications and approval of rivaroxaban.

\begin{tabular}{lcc}
\hline Indication & Year of approval & Country \\
\hline DVT/PE prophylaxis in hip and knee surgery & 2008 in Europe \\
2011 in USA & 2011 & USA and Europe \\
Atrial fibrillation & 2012 & USA and Europe \\
DVT/PE treatment & 2013 & Europe \\
Acute coronary syndrome & 2017 & USA and Europe \\
To reduce risk of VTE after 6 months of treatment of DVT/PE & 2018 & USA and Europe \\
Stable CAD & 2018 & USA and Europe \\
PAD & 2019 & USA \\
DVT/PE prophylaxis in acute medical illness & & \\
\hline
\end{tabular}

DVT/PE: deep vein thrombosis/pulmonary embolism; CAD: coronary artery disease; PAD: peripheral arterial disease.

10-14 days to enoxaparin $30 \mathrm{mg}$ twice daily for 10-14 days [26-29]. All these trials demonstrated the superiority of rivaroxaban over enoxaparin without increasing the risk of bleeding [22-25]. These trials led to FDA approval of rivaroxaban $10 \mathrm{mg}$ daily after hip and knee surgery in 2012 [30]. In the ninth edition of American College of Chest Physicians (ACCP) guidelines, rivaroxaban was recommended to be considered after hip and knee arthroplasty as an alternative to enoxaparin [31].

A prophylactic dose of rivaroxaban was later compared to low-dose aspirin in the EPCAT II (Extended Venous Thromboembolism Prophylaxis Comparing Rivaroxaban to Aspirin Following Total Hip and Knee Arthroplasty II) trial. In this trial, all patients after knee and hip arthroplasty were treated with rivaroxaban for five days and then were divided into two groups. One group received aspirin $81 \mathrm{mg}$ daily, and the second group received rivaroxaban $10 \mathrm{mg}$ daily for nine days after knee replacement and 30 days after hip replacement. After short postoperative prophylaxis of rivaroxaban, aspirin was noninferior to rivaroxaban in preventing venous thromboembolism [32]. The systematic review and metaanalysis of randomized controlled trials evaluating the safety and efficacy of rivaroxaban after total hip and knee replacement found that rivaroxaban is safe and effective with a low incidence of thrombotic and bleeding events [24].

\section{Venous Thromboembolism Prophylaxis in Acute Medical Illness}

Rivaroxaban was studied in the MAGELLAN (Multicenter, Randomized, Parallel-Group Efficacy and Safety for the Prevention of Venous Thromboembolism in Hospitalized Acutely Ill Medical Patients) trial to check the appropriate duration of anticoagulation in these sick patients to prevent VTE [33]. The rivaroxaban group received $10 \mathrm{mg}$ daily dose for $35 \pm 4$ days with a placebo subcutaneous injection for 10 \pm 4 days while the enoxaparin group received $40 \mathrm{mg}$ daily subcutaneous enoxaparin for $10 \pm 4$ days and an oral placebo for $35 \pm 4$ days. The efficacy of standard duration rivaroxaban (10 \pm 4 days) was like that of enoxaparin, whereas the effectiveness of extended duration ( $35 \pm 4$ days) rivaroxaban was superior to enoxaparin. However, rivaroxaban was associated with more negative safety outcomes of clinically relevant bleeding events [31].

Rivaroxaban was also compared to a placebo after hospital discharge for 45 days in the MARINER (Medically Ill Patient Assessment of Rivaroxaban versus Placebo in Reducing Post-Discharge Venous Thrombo-Embolism Risk) trial. However, the results of this trial did not indicate positive health outcomes from the administration of rivaroxaban. Rivaroxaban was not associated with a significantly lower risk of symptomatic VTE and death due to VTE as compared to placebo [34].

Most studies indicated positive health outcomes for those who took rivaroxaban, but some negative and some nonsuperior effects were also found. The FDA approved rivaroxaban to prevent VTE in acutely ill patients in October 2019, and based on this approval, rivaroxaban can be initiated during hospitalizations and continued for 31-39 days [35]. In the systematic review and meta-analysis comparing prolonged thromboprophylaxis with factor Xa inhibitors to the shortterm enoxaparin, DOACs were more effective than enoxaparin but were also associated with more bleeding episodes [36].

\section{Role in Peripheral Artery Disease and Chronic Coronary Artery Disease}

Patients with established cardiovascular disease remain at high risk for recurrent cardiovascular events [37]. This led to the landmark COMPASS (Cardiovascular Outcomes for People Using Anticoagulant Strategies) trial, which hypothesized that rivaroxaban with aspirin or alone is more effective than aspirin in preventing recurrent cardiovascular events and is safe in patients with stable atherosclerotic vascular disease [38]. In this randomized trial, it was found that in patients with stable atherosclerotic vascular disease, rivaroxaban $2.5 \mathrm{mg}$ twice daily plus aspirin $100 \mathrm{mg}$ once daily was associated with significantly lower major adverse cardiovascular events (MACE), but significantly higher major bleeding than with aspirin alone. Rivaroxaban $5 \mathrm{mg}$ twice daily alone did not result in a significantly lower risk of MACE but had a significantly higher risk of major bleeding as compared to aspirin alone [34]. 
Based on this landmark clinical trial, the FDA approved rivaroxaban $2.5 \mathrm{mg}$ twice daily with aspirin $75-100 \mathrm{mg}$ daily in patients with stable coronary artery disease and peripheral artery disease. Although there were more bleeding episodes in the COMPASS trial, there were no intracranial or fatal bleeding events. Based on this, the author is of the opinion that shared decision making should be adopted to prescribe low-dose rivaroxaban $2.5 \mathrm{mg}$ twice daily with aspirin $81 \mathrm{mg}$ daily [39].

In the systematic review and meta-analysis on the use of rivaroxaban in coronary artery disease, it was concluded that patient's characteristics of ischemic and bleeding risk are critical in deciding about the addition of rivaroxaban [40]. The low-dose rivaroxaban in peripheral arterial disease was studied in the systematic review of randomized trials, and it was found to be effective in preventing cardiovascular events with a possible increase risk of bleeding [41].

\section{Use in Acute Coronary Disease}

Patients with acute coronary syndrome manifest increased coagulation system activity in the acute phase which persists even beyond the acute phase [42]. The ATLAS ACS-TIMI 46 (Anti-Xa Therapy to Lower Cardiovascular Events in Addition to Standard Therapy in Subjects with Acute Coronary Syndrome-Thrombolysis in Myocardial Infarction 46) phase II trial was a dose-finding trial. Rivaroxaban 5-20 mg daily total doses in recent acute coronary syndrome were found to reduce major adverse cardiovascular events with dosedependent increased bleeding events [43]. This led to a phase III trial, ATLAS ACS 2-TIMI 51, which was designed to determine the clinically effective dose of rivaroxaban in recent acute coronary syndrome. In this study, rivaroxaban $2.5 \mathrm{mg}$ twice daily and $5 \mathrm{mg}$ twice daily reduced the primary efficacy endpoint of death from a cardiovascular cause, myocardial infarction, and stroke as compared to placebo. However, there were significantly more adverse events of bleeding with both doses, although the 2.5 dosage had fewer bleeding events than the $5 \mathrm{mg}$ [44]. The direct oral anticoagulants studied in systemic review and meta-analysis were found to have a modest beneficial effect in acute coronary syndrome with an increased risk of bleeding, and shared decision should be made in the use of DOACs in these patients [45].

\section{Role in Treatment of Venous Thromboembolism with Cancer}

VTE is common in patients with cancer, and subcutaneous low molecular weight heparin is the standard therapy in these patients [46]. Therapeutic dose of rivaroxaban was studied in SELECT-D (Anticoagulation Therapy in Selected Cancer Patients at Risk of Recurrence of Venous Thromboembolism), a randomized, multicenter pilot trial, and was compared to the therapeutic dose of dalteparin in patients with active cancer and diagnosis of VTE. There was less cumulative VTE recurrence in the rivaroxaban group compared to the dalteparin group [40]. Based on this trial, it was concluded that rivaroxaban at standard DVT/PE dosage could be considered in patients with active cancer and diagnosis of VTE after shared decision making. The use of DOACs in VTE in cancer patients was found to be as safe and effective as conventional therapy in the systematic review and metaanalysis of 10 studies comparing DOACs with conventional therapy [47].

\section{Use after Transcatheter Aortic Valve Replacement (TAVR)}

In the current 2014 guidelines by ACC/AHA on antithrombotic regimen after transcatheter aortic valve replacement (TAVR), clopidogrel $75 \mathrm{mg}$ daily for the first six months after TAVR has a class IIb recommendation with the level of evidence (LOE) C, along with lifelong aspirin $75 \mathrm{mg}$ daily after TAVR [48]. Because of the reported concern of subclinical TAVR valve thrombosis, rivaroxaban was studied in the GALILEO (Global Study Comparing a Rivaroxaban-based Antithrombotic Strategy after Transcatheter Aortic Valve Replacement to Optimize Clinical Outcomes) trial [49]. This trial compared rivaroxaban $10 \mathrm{mg}$ daily with aspirin 75 $100 \mathrm{mg}$ daily in the first three months after TAVR to aspirin $75-100 \mathrm{mg}$ daily with clopidogrel $75 \mathrm{mg}$ daily in the first three months after TAVR. It found that rivaroxaban was associated with higher mortality, thromboembolic complications, and bleeding events compared to the antiplatelet regimen and was terminated early at a median follow-up of 17 months [42]. The use of rivaroxaban is not recommended in prosthetic heart valves. There is also a study being conducted in Brazil called the RIWA (Rivaroxaban vs. Warfarin in Patients with Mechanical Heart Valves) trial. This phase II/III prospective randomized trial is aimed at evaluating the efficacy and safety of rivaroxaban $15 \mathrm{mg}$ twice daily vs. warfarin in mechanical prosthetic valves [50].

\section{Use in Heart Failure with Reduced Ejection Fraction}

Heart failure is considered a hypercoagulable state, and patients with acute or chronic heart failure are at increased risk for thrombotic events, including coronary thrombosis, intraventricular thrombosis, and systemic embolism [51]. The COMMANDER HF (A Study to Assess the Effectiveness and Safety of Rivaroxaban in Reducing the Risk of Death, Myocardial Infarction, or Stroke in Participants with Heart Failure and Coronary Artery Disease Following an Episode of Decompensated Heart Failure) trial was conducted with rivaroxaban $2.5 \mathrm{mg}$ twice daily compared to a placebo in patients with median ejection fraction (EF) of $35 \%$ in the rivaroxaban group and $34 \%$ in placebo. This was a negative trial: rivaroxaban in patients with recent worsening of chronic heart failure with low EF, and coronary artery disease, was not associated with a reduction in the composite outcome of all-cause mortality, myocardial infarction, or stroke than placebo. Additionally, it did not favorably influence rehospitalization for heart failure [52]. The same outcomes were demonstrated in systematic review and meta-analysis on anticoagulation that rivaroxaban did not reduce mortality, 
heart failure readmission, or MI in patients with low $\mathrm{EF}$ and normal sinus rhythm [53].

\section{Role in Treatment of Left Ventricular (LV) Thrombus}

DOACs have been used off label in LV thrombus, but the data is limited [54]. A three-center cohort study performed on 514 patients with LV thrombus showed that DOACs are associated with higher rates of stroke and systemic embolism than warfarin in a retrospective analysis. It is also interesting to note that patients even with the resolution of LV thrombus on echocardiography still experienced a stroke and systemic embolism [55].

\section{Use after Left Atrial Appendage Occlusion (LAAO)}

LAAO is performed in patients who have nonvalvular atrial fibrillation and cannot take anticoagulants or do not wish to be on anticoagulants [3]. In the landmark clinical trials of LAAO, warfarin was used transiently postoperatively. However, in the real world, there is an increased use of DOACs, but no randomized studies are being conducted on the transient use of DOACs after LAAO [56].

\section{Antiphospholipid Syndrome (APS)}

APS in association with persistent antiphospholipid antibodies of lupus anticoagulant, anticardiolipin antibodies, and/or anti-beta 2 glycoprotein antibodies manifests with arterial and venous thrombosis [57]. Rivaroxaban was studied in thrombotic APS in a randomized noninferiority trial and did not show noninferiority to dose-adjusted warfarin. It also showed a statistically nonsignificant doubling of the recurrent thrombotic events [58].

\section{Consideration in Kidney Disease}

In the ROCKET AF trial, patients with $\mathrm{CrCl}<30 \mathrm{~mL} / \mathrm{min}$ and end-stage renal disease (ESRD) were not included, but rivaroxaban is approved by the FDA with a reduced dose of $15 \mathrm{mg}$ daily with $\mathrm{CrCl} 15-50 \mathrm{~mL} / \mathrm{min}[6,7]$. This is not recommended in patients with ESRD and atrial fibrillation, and a recent study using Medicare fee-for-service 5\% claims data from 2007 to 2013 analyzed treatment and outcomes in patients with atrial fibrillation and ESRD [59]. There was less use of oral anticoagulation in patients with AF and ESRD, and the use of anticoagulation (VKA, apixaban, rivaroxaban, and dabigatran) was not associated with reduced stroke or death but was associated with increased risk of hospitalization for bleeding or intracranial hemorrhage [51]. The use of rivaroxaban in AF was also associated with a higher risk of bleeding in AF patients on hemodialysis in a systemic review of the randomized trials, cohort studies, and case series [60]. Rivaroxaban was not studied in the treatment or prophylaxis of $\mathrm{DVT} / \mathrm{PE}$ in patients with $\mathrm{CrCl}<30 \mathrm{~mL} / \mathrm{min}$, while the reduced dose of $2.5 \mathrm{mg}$ twice daily was not tested in patients with $\mathrm{CrCl}<15 \mathrm{~mL} / \mathrm{min}[16,17,30,31,35]$. Rivaroxaban is not recommended for the treatment of DVT/PE if $\mathrm{CrCl}$ is $<30 \mathrm{~mL} / \mathrm{min}$, while the $2.5 \mathrm{mg}$ twice daily dose should be used with caution in coronary artery disease and peripheral artery disease with $\mathrm{CrCl} 15-30 \mathrm{~mL} / \mathrm{min}$ and is not recommended in ESRD [16, 17, 30, 31, 35]. The dosage of rivaroxaban in various indications and different glomerular filtration rate is shown in Table 2.

\section{Consideration in Liver Disease}

No clinical data is available for the use of rivaroxaban in patients with severe hepatic impairment, and its use is prohibited in Child-Pugh B and C class or any impairment associated with coagulopathy [61].

\section{Consideration in Obesity}

No large randomized controlled trials have specifically investigated the efficacy and safety of DOACs in the obese population [62]. The International Society of Thrombosis and Hemostasis in their recent guidelines of 2016 recommended against the use of DOACs in extremely obese patients with weight $>120 \mathrm{~kg}$ or body mass index $>40 \mathrm{~kg} / \mathrm{m}^{2}$. However, if for some reasons DOACs are used in these patients, then the society recommended checking drug-specific antifactor levels, like antifactor Xa, with the use of rivaroxaban [52].

\section{Cost Economic Analysis}

The cost economic analysis of rivaroxaban for various indications and in different countries is reported in literature. Rivaroxaban is found to be cost-effective and is briefly discussed herein based on the literature search in a few countries. In the Netherlands, rivaroxaban was found to be cost-effective for the treatment and secondary prevention of venous thromboembolism with health gains and cost savings of $€ 304$ over the patient's life compared to LMWH/warfarin [63]. The Brazilian experience also demonstrated cost-effectiveness of rivaroxaban compared to warfarin in the management of venous thromboembolism [64]. Rivaroxaban was also found to be cost-effective in elective electrical cardioversion compared to warfarin in the Netherlands with a $50 \%$ probability of being cost-effective compared to warfarin [65]. In Greece, rivaroxaban was also cost-effective in the management of DVT/PE [66]. Rivaroxaban was also found to cost-effective in the treatment of venous thromboembolism in the Chinese population when compared to warfarin [67]. In the elderly population in the US with worsening renal function, rivaroxaban was found to be cost-effective compared to warfarin for the treatment of nonvalvular atrial fibrillation [68]. In the treatment of PAD when clinic-economic analysis of rivaroxaban compared to clopidogrel was performed, rivaroxaban was found to be cost-effective with a saving of 682 US dollars per participant [39]. Rivaroxaban was found to be costeffective in the management of cancer-associated thrombosis when compared to dalteparin in the US population [69]. The cost of stable atherosclerotic disease was analyzed in the Australian population; rivaroxaban with aspirin was found to be cost-effective compared to aspirin alone [70]. Rivaroxaban 
TABLE 2: Rivaroxaban dosage in various indications.

\begin{tabular}{|c|c|c|}
\hline Indication & GFR & Dose \\
\hline \multirow{3}{*}{ Nonvalvular AFib } & $>50 \mathrm{~mL} / \mathrm{min}$ & 20 mg daily \\
\hline & $15-50 \mathrm{~mL} / \mathrm{min}$ & $15 \mathrm{mg}$ daily \\
\hline & $<15 \mathrm{~mL} / \mathrm{min}$ & Not recommended \\
\hline \multirow{2}{*}{ Treatment of DVT/PE } & $>30 \mathrm{~mL} / \mathrm{min}$ & $15 \mathrm{mg}$ BID for 3 weeks followed by $20 \mathrm{mg}$ daily. \\
\hline & $<30 \mathrm{~mL} / \mathrm{min}$ & Not recommended \\
\hline \multirow{2}{*}{ DVT/PE prophylaxis in hip and knee surgery } & $>30 \mathrm{~mL} / \mathrm{min}$ & $10 \mathrm{mg}$ daily \\
\hline & $<30 \mathrm{~mL} / \mathrm{min}$ & Not recommended \\
\hline \multirow{2}{*}{ DVT/PE prophylaxis in acute medical illness } & $>30 \mathrm{~mL} / \mathrm{min}$ & $10 \mathrm{mg}$ daily \\
\hline & $<30 \mathrm{~mL} / \mathrm{min}$ & Not recommended \\
\hline \multirow{3}{*}{ Stable coronary artery disease } & $>30 \mathrm{~mL} / \mathrm{min}$ & $2.5 \mathrm{mg}$ BID \\
\hline & $15-30 \mathrm{~mL} / \mathrm{min}$ & $2.5 \mathrm{mg}$ BID with caution \\
\hline & $<15 \mathrm{~mL} / \mathrm{min}$ & Not recommended \\
\hline \multirow{3}{*}{ Peripheral artery disease } & $>30 \mathrm{~mL} / \mathrm{min}$ & $2.5 \mathrm{mg}$ BID \\
\hline & $15-30 \mathrm{~mL} / \mathrm{min}$ & $2.5 \mathrm{mg}$ BID with caution \\
\hline & $<15 \mathrm{~mL} / \mathrm{min}$ & Not recommended \\
\hline \multirow{3}{*}{ Acute coronary artery syndrome } & $>30 \mathrm{~mL} / \mathrm{min}$ & $2.5 \mathrm{BID}$ \\
\hline & $15-30 \mathrm{~mL} / \mathrm{min}$ & $2.5 \mathrm{mg}$ BID with caution \\
\hline & $<15 \mathrm{~mL} / \mathrm{min}$ & Not recommended \\
\hline
\end{tabular}

GFR: glomerular filtration rate; BID: twice daily; AFib: atrial fibrillation; DVT/PE: deep vein thrombosis/pulmonary embolism.

was also found to be cost-effective compared to enoxaparin for the prevention of venous thromboembolism after hip and knee replacement surgeries [71].

\section{Safety}

Bleeding is one of the main side effects of rivaroxaban, and it can vary from minor bruising to major life-threatening bleeding. Intracranial, gastrointestinal, and other intracavitary bleeding events are reported with rivaroxaban, although less frequently than with warfarin [72]. Based on the systematic review and metanalysis of randomized trials comparing the risk of gastrointestinal bleeding in patients taking direct oral anticoagulants, rivaroxaban and dabigatran were associated with increased gastrointestinal bleeding compared to other direct oral anticoagulants [73]. These bleeding events usually occur in patients with other comorbid conditions like hypertension, abnormal liver and renal functions, old age, concomitant use of other blood thinners, and previous episodes of bleeding [74].

Another potential limitation is premature discontinuation of this drug as it is associated with higher risk of thrombosis and is strongly discouraged because of this reason [75, 76]. As rivaroxaban can increase bleeding tendency in various invasive procedures, although there are no definite guidelines, rivaroxaban should be stopped for about two half-lives before spinal puncture/anesthesia to prevent spinal hematoma $[75,76]$.

Rivaroxaban has several drug interactions and should not be used with P-glycoprotein and CYP3A inhibitors or inducers because of the variability in the efficacy of rivaroxaban and increased risk of thrombosis or bleeding [77].
Liver injury, hypersensitivity reactions, leukocytoclastic vasculitis, and hair loss are nonhemorrhagic but rare side effects of rivaroxaban reported in literature [78].

\section{Rivaroxaban Reversal Agent}

The factor Xa reversal, andexanet alpha, which is a modified recombinant inactive form of human factor $\mathrm{Xa}$, was studied in ANNEXA-4 (Andexanet Alpha, A Novel Antidote to the Anticoagulation Effects of Factor Xa Inhibitors), a single group cohort study [79]. In this study, it was found that in patients with major acute bleeding associated with the use of factor Xa inhibitor, treatment with andexanet significantly reduced factor Xa activity, and $82 \%$ of the patients had an excellent or good hemostatic efficacy at 12 hours. Andexanet alpha was approved by the FDA in May 2018 [80]. It is available in low and high doses. A low dose is $400 \mathrm{mg}$ intravenous bolus at the rate of $30 \mathrm{mg} / \mathrm{minute}$ followed by $4 \mathrm{mg} / \mathrm{hour}$ for 2 hours for patients taking $\leq 10 \mathrm{mg}$ daily rivaroxaban or if $>10 \mathrm{mg}$ daily dose with the last dose taken $>8$ hours before the administration of andexanet. The high dose is $800 \mathrm{mg}$ intravenous bolus at the rate of $30 \mathrm{mg} / \mathrm{minute}$ followed by $8 \mathrm{mg} /$ hour for 2 hours for rivaroxaban dose $>10 \mathrm{mg}$ with the last dose hours before the administration of andexanet $<8$ hours.

\section{Conclusion}

Rivaroxaban has been studied in various indications and has robust positive data in numerous cardiovascular indications, including nonvalvular atrial fibrillation, treatment, and prophylaxis of deep venous thrombosis and pulmonary 
embolism, peripheral artery disease, stable coronary artery disease, acute coronary syndrome, and venous thromboembolism in cancer patients. This data is supported by observational studies, randomized trials, systematic review, and meta-analysis. Rivaroxaban is also proved to be costeffective worldwide compared to standard of care therapies in various indications.

On the other hand, the randomized clinical trials of rivaroxaban in the transcatheter aortic valve, thrombotic antiphospholipid syndrome, and heart failure with reduced ejection fraction and observational cohort study in left ventricular thrombus were negative, and rivaroxaban should not be used in these patients.

\section{Conflicts of Interest}

The authors declare that they have no conflicts of interest.

\section{References}

[1] W. Mueck, J. Stampfuss, D. Kubitza, and M. Becka, "Clinical pharmacokinetic and pharmacodynamic profile of rivaroxaban," Clinical Pharmacokinetics, vol. 53, no. 1, pp. 1-16, 2014.

[2] K. P. Cabral and J. E. Ansell, "The role of factor Xa inhibitors in venous thromboembolism treatment," Vascular Health and Risk Management, vol. 11, pp. 117-123, 2015.

[3] M. Ajmal, H. Naik, and A. Kocheril, "Left atrial appendage closure in patients with intracranial hemorrhage and nonvalvular atrial fibrillation," Journal of Stroke and Cerebrovascular Diseases, vol. 29, no. 4, p. 104685, 2020.

[4] J. Amerena and D. Ridley, "An update on anticoagulation in atrial fibrillation," Heart, Lung \& Circulation, vol. 26, no. 9, pp. 911-917, 2017.

[5] V. Mtwesi and G. Amit, "Stroke prevention in atrial fibrillation," The Medical Clinics of North America, vol. 103, no. 5, pp. 847-862, 2019.

[6] M. R. Patel, K. W. Mahaffey, J. Garg et al., "Rivaroxaban versus warfarin in nonvalvular atrial fibrillation," The New England Journal of Medicine, vol. 365, no. 10, pp. 883-891, 2011.

[7] N. Jain and R. F. Reilly, "Clinical pharmacology of oral anticoagulants in patients with kidney disease," Clinical Journal of the American Society of Nephrology, vol. 14, no. 2, pp. 278287, 2019.

[8] K. Vimalesvaran, S. J. Dockrill, and D. A. Gorog, "Role of rivaroxaban in the management of atrial fibrillation: insights from clinical practice," Vascular Health and Risk Management, vol. Volume 14, pp. 13-21, 2018.

[9] G. Ntaios, V. Papavasileiou, K. Makaritsis, K. Vemmos, P. Michel, and G. Y. H. Lip, "Real-world setting comparison of nonvitamin- $\mathrm{K}$ antagonist oral anticoagulants versus vitamin-K antagonists for stroke prevention in atrial fibrillation: a systematic review and meta-analysis," Stroke, vol. 48, no. 9, pp. 2494-2503, 2017.

[10] J. A. López-López, J. A. Sterne, H. H. Thom et al., "Oral anticoagulants for prevention of stroke in atrial fibrillation: systematic review, network meta-analysis, and cost effectiveness analysis," BMJ, vol. 359, p. j5058, 2017.

[11] C. T. January, L. S. Wann, H. Calkins et al., “2019 AHA/ACC/HRS focused update of the 2014 AHA/ACC/HRS guideline for the management of patients with atrial fibrillation: a report of the American College of Cardiology/American
Heart Association task force on clinical practice guidelines and the Heart Rhythm Society," Journal of the American College of Cardiology, vol. 74, no. 1, pp. 104-132, 2019.

[12] C. T. January, L. S. Wann, J. S. Alpert et al., "2014 AHA/ACC/HRS guideline for the management of patients with atrial fibrillation: a report of the American College of Cardiology/American Heart Association task force on practice guidelines and the Heart Rhythm Society," Journal of the American College of Cardiology, vol. 64, no. 21, pp. e1-76, 2014.

[13] M. Olaf and R. Cooney, "Deep venous thrombosis," Emergency Medicine Clinics of North America, vol. 35, no. 4, pp. 743-770, 2017.

[14] E. O. Essien, P. Rali, and S. C. Mathai, "Pulmonary embolism," The Medical Clinics of North America, vol. 103, no. 3, pp. 549564, 2019.

[15] G. Agnelli, A. Gallus, S. Z. Goldhaber et al., "Treatment of proximal deep-vein thrombosis with the oral direct factor Xa inhibitor rivaroxaban (BAY 59-7939): the ODIXa-DVT (oral direct factor Xa inhibitor BAY 59-7939 in patients with acute symptomatic deep-vein thrombosis) study," Circulation, vol. 116, no. 2, pp. 180-187, 2007.

[16] H. R. Buller, A. W. A. Lensing, M. H. Prins et al., "A doseranging study evaluating once-daily oral administration of the factor Xa inhibitor rivaroxaban in the treatment of patients with acute symptomatic deep vein thrombosis: the EinsteinDVT dose-ranging study," Blood, vol. 112, no. 6, pp. $2242-$ 2247, 2008.

[17] The EINSTEIN Investigators, "Oral rivaroxaban for symptomatic venous thromboembolism," The New England Journal of Medicine, vol. 363, no. 26, pp. 2499-2510, 2010.

[18] The EINSTEIN-PE Investigators, "Oral rivaroxaban for the treatment of symptomatic pulmonary embolism," The New England Journal of Medicine, vol. 366, no. 14, pp. 1287-1297, 2012.

[19] A. Reis, "Prevention and treatment of venous thromboembolism: the place of new oral anticoagulants," Revista Portuguesa de Cardiologia: Orgao Oficial da Sociedade Portuguesa de Cardiologia = Portuguese Journal of Cardiology: an Official Journal of the Portuguese Society of Cardiology, vol. 31, Supplement 1, pp. 45-50, 2012.

[20] J. I. Weitz, A. W. A. Lensing, M. H. Prins et al., "Rivaroxaban or aspirin for extended treatment of venous thromboembolism," The New England Journal of Medicine, vol. 376, no. 13, pp. 1211-1222, 2017.

[21] S. Barco, I. Schmidtmann, W. Ageno et al., "Early discharge and home treatment of patients with low-risk pulmonary embolism with the oral factor Xa inhibitor rivaroxaban: an international multicentre single-arm clinical trial," European Heart Journal, vol. 41, no. 4, pp. 509-518, 2020.

[22] W. Frank Peacock, C. I. Coleman, D. B. Diercks et al., "Emergency department discharge of pulmonary embolus patients," Academic Emergency Medicine, vol. 25, no. 9, pp. 995-1003, 2018.

[23] A. T. Cohen, M. Hamilton, S. A. Mitchell et al., "Comparison of the novel oral anticoagulants apixaban, dabigatran, edoxaban, and rivaroxaban in the initial and long-term treatment and prevention of venous thromboembolism: systematic review and network meta-analysis," PLoS One, vol. 10, no. 12, article e0144856, 2015.

[24] J. Liu, J. Zhao, Y. Yan, and J. Su, "Effectiveness and safety of rivaroxaban for the prevention of thrombosis following total 
hip or knee replacement: a systematic review and meta-analysis," Medicine (Baltimore), vol. 98, no. 9, article e14539, 2019.

[25] W. H. Geerts, G. F. Pineo, J. A. Heit et al., "Prevention of venous thromboembolism: the Seventh ACCP Conference on Antithrombotic and Thrombolytic Therapy," Chest, vol. 126, no. 3, pp. 338s-400s, 2004.

[26] B. I. Eriksson, L. C. Borris, R. J. Friedman et al., "Rivaroxaban versus enoxaparin for thromboprophylaxis after hip arthroplasty," The New England Journal of Medicine, vol. 358, no. 26, pp. 2765-2775, 2008.

[27] M. R. Lassen, W. Ageno, L. C. Borris et al., "Rivaroxaban versus enoxaparin for thromboprophylaxis after total knee arthroplasty," The New England Journal of Medicine, vol. 358, no. 26, pp. 2776-2786, 2008.

[28] A. G. Turpie, M. R. Lassen, B. L. Davidson et al., "Rivaroxaban versus enoxaparin for thromboprophylaxis after total knee arthroplasty (RECORD4): a randomised trial," Lancet, vol. 373, no. 9676, pp. 1673-1680, 2009.

[29] A. K. Kakkar, B. Brenner, O. E. Dahl et al., "Extended duration rivaroxaban versus short-term enoxaparin for the prevention of venous thromboembolism after total hip arthroplasty: a double-blind, randomised controlled trial," Lancet, vol. 372, no. 9632, pp. 31-39, 2008.

[30] P. D. Stein, F. Matta, and M. J. Hughes, "Home treatment of deep venous thrombosis according to comorbid conditions," The American Journal of Medicine, vol. 129, no. 4, pp. 392397, 2016.

[31] Y. Falck-Ytter, C. W. Francis, N. A. Johanson et al., "Prevention of VTE in orthopedic surgery patients: antithrombotic therapy and prevention of thrombosis, 9th ed: American College of Chest Physicians Evidence-Based Clinical Practice Guidelines," Chest, vol. 141, no. 2, pp. e278S-e325S, 2012.

[32] D. R. Anderson, M. Dunbar, J. Murnaghan et al., "Aspirin or rivaroxaban for VTE prophylaxis after hip or knee arthroplasty," The New England Journal of Medicine, vol. 378, no. 8, pp. 699-707, 2018.

[33] A. T. Cohen, T. E. Spiro, H. R. Büller et al., "Rivaroxaban for thromboprophylaxis in acutely ill medical patients," The New England Journal of Medicine, vol. 368, no. 6, pp. 513-523, 2013.

[34] A. C. Spyropoulos, W. Ageno, G. W. Albers et al., "Rivaroxaban for thromboprophylaxis after hospitalization for medical illness," The New England Journal of Medicine, vol. 379, no. 12, pp. 1118-1127, 2018.

[35] K. MacDougall and A. C. Spyropoulos, "New paradigms of extended thromboprophylaxis in medically ill patients," Journal of Clinical Medicine, vol. 9, no. 4, p. 1002, 2020.

[36] I. E. Albertsen, T. B. Larsen, L. H. Rasmussen, T. F. Overvad, and G. Y. H. Lip, "Prevention of venous thromboembolism with new oral anticoagulants versus standard pharmacological treatment in acute medically ill patients: a systematic review and meta-analysis," Drugs, vol. 72, no. 13, pp. 1755-1764, 2012.

[37] D. L. Bhatt, K. A. Eagle, E. M. Ohman et al., "Comparative determinants of 4-year cardiovascular event rates in stable outpatients at risk of or with atherothrombosis," JAMA, vol. 304, no. 12, pp. 1350-1357, 2010.

[38] J. W. Eikelboom, S. J. Connolly, J. Bosch et al., "Rivaroxaban with or without aspirin in stable cardiovascular disease," The New England Journal of Medicine, vol. 377, no. 14, pp. 13191330, 2017.
[39] D. I. Tsilimigras, D. Moris, G. Karaolanis, S. K. Kakkos, K. Filis, and F. Sigala, "Rivaroxaban versus clopidogrel for peripheral artery disease: a clinico-economic approach of the COMPASS trial," Current Pharmaceutical Design, vol. 24, no. 38, pp. 4516-4517, 2018.

[40] C. Xie, Y. Hang, J. Zhu et al., "Benefit and risk of adding rivaroxaban in patients with coronary artery disease: a systematic review and meta-analysis," Clinical Cardiology, 2020.

[41] R. Bauersachs, O. Wu, J. B. Briere et al., "Antithrombotic treatments in patients with chronic coronary artery disease or peripheral artery disease: a systematic review of randomised controlled trials," Cardiovascular Therapeutics, vol. 2020, Article ID 3057168, 11 pages, 2020.

[42] P. A. Merlini, K. A. Bauer, L. Oltrona et al., "Persistent activation of coagulation mechanism in unstable angina and myocardial infarction," Circulation, vol. 90, no. 1, pp. 61-68, 1994.

[43] J. L. Mega, E. Braunwald, S. Mohanavelu et al., "Rivaroxaban versus placebo in patients with acute coronary syndromes (ATLAS ACS-TIMI 46): a randomised, double-blind, phase II trial," Lancet, vol. 374, no. 9683, pp. 29-38, 2009.

[44] J. L. Mega, E. Braunwald, S. D. Wiviott et al., "Rivaroxaban in patients with a recent acute coronary syndrome," The New England Journal of Medicine, vol. 366, no. 1, pp. 9-19, 2012.

[45] J. Oldgren, L. Wallentin, J. H. Alexander et al., "New oral anticoagulants in addition to single or dual antiplatelet therapy after an acute coronary syndrome: a systematic review and meta-analysis," European Heart Journal, vol. 34, no. 22, pp. 1670-1680, 2013.

[46] A. M. Young, A. Marshall, J. Thirlwall et al., "Comparison of an oral factor Xa inhibitor with low molecular weight heparin in patients with cancer with venous thromboembolism: results of a randomized trial (SELECT-D)," Journal of Clinical Oncology, vol. 36, no. 20, pp. 2017-2023, 2018.

[47] M. C. Vedovati, F. Germini, G. Agnelli, and C. Becattini, "Direct oral anticoagulants in patients with VTE and cancer: a systematic review and meta-analysis," Chest, vol. 147, no. 2, pp. 475-483, 2015.

[48] R. A. Nishimura, C. M. Otto, R. O. Bonow et al., "2014 AHA/ACC guideline for the management of patients with valvular heart disease: executive summary: a report of the American College of Cardiology/American Heart Association task force on practice guidelines," Circulation, vol. 129, no. 23, pp. 2440-2492, 2014.

[49] G. D. Dangas, J. G. P. Tijssen, J. Wöhrle et al., “A controlled trial of rivaroxaban after transcatheter aortic-valve replacement," The New England Journal of Medicine, vol. 382, no. 2, pp. 120-129, 2020.

[50] A. R. Durães, Y. de Souza Lima Bitar, J. A. L. Filho et al., "Rivaroxaban versus warfarin in patients with mechanical heart valve: rationale and design of the RIWA study," Drugs in ReD, vol. 18, no. 4, pp. 303-308, 2018.

[51] F. Zannad, W. G. Stough, V. Regnault et al., "Is thrombosis a contributor to heart failure pathophysiology? Possible mechanisms, therapeutic opportunities, and clinical investigation challenges," International Journal of Cardiology, vol. 167, no. 5, pp. 1772-1782, 2013.

[52] F. Zannad, S. D. Anker, W. M. Byra et al., "Rivaroxaban in patients with heart failure, sinus rhythm, and coronary disease," The New England Journal of Medicine, vol. 379, no. 14, pp. 1332-1342, 2018. 
[53] E. M. Sulaica, T. E. Macaulay, R. R. Helbing, M. Abo-Aly, A. Abdel-Latif, and M. A. Wanat, "A comparison of anticoagulation, antiplatelet, and placebo treatment for patients with heart failure reduced ejection fraction in sinus rhythm: a systematic review and meta-analysis," Heart Failure Reviews, vol. 25, no. 2, pp. 207-216, 2020.

[54] A. M. Fleddermann, C. H. Hayes, A. Magalski, and M. L. Main, "Efficacy of direct acting oral anticoagulants in treatment of left ventricular thrombus," The American Journal of Cardiology, vol. 124, no. 3, pp. 367-372, 2019.

[55] A. A. Robinson, C. R. Trankle, G. Eubanks et al., "Off-label use of direct oral anticoagulants compared with warfarin for left ventricular thrombi," JAMA Cardiology, vol. 5, no. 6, pp. 685-692, 2020.

[56] L. I. Bösche, F. Afshari, D. Schöne, A. Ewers, A. Mügge, and M. Gotzmann, "Initial experience with novel oral anticoagulants during the first 45 days after left atrial appendage closure with the watchman device," Clinical Cardiology, vol. 38, no. 12, pp. 720-724, 2015.

[57] H. Cohen, M. Efthymiou, and D. A. Isenberg, "Use of direct oral anticoagulants in antiphospholipid syndrome," Journal of Thrombosis and Haemostasis, vol. 16, no. 6, pp. 10281039, 2018.

[58] J. Ordi-Ros, L. Sáez-Comet, M. Pérez-Conesa et al., "Rivaroxaban versus vitamin $\mathrm{K}$ antagonist in antiphospholipid syndrome: a randomized noninferiority trial," Annals of Internal Medicine, vol. 171, no. 10, pp. 685-694, 2019.

[59] S. D. Pokorney, E. Black-Maier, A. S. Hellkamp et al., "Oral anticoagulation and cardiovascular outcomes in patients with atrial fibrillation and end-stage renal disease," Journal of the American College of Cardiology, vol. 75, no. 11, pp. 12991308, 2020.

[60] J. Feldberg, P. Patel, A. Farrell et al., "A systematic review of direct oral anticoagulant use in chronic kidney disease and dialysis patients with atrial fibrillation," Nephrology, Dialysis, Transplantation, vol. 34, no. 2, pp. 265-277, 2019.

[61] J. Graff and S. Harder, "Anticoagulant therapy with the oral direct factor Xa inhibitors rivaroxaban, apixaban and edoxaban and the thrombin inhibitor dabigatran etexilate in patients with hepatic impairment," Clinical Pharmacokinetics, vol. 52, no. 4, pp. 243-254, 2013.

[62] K. Martin, J. Beyer-Westendorf, B. L. Davidson, M. V. Huisman, P. M. Sandset, and S. Moll, "Use of the direct oral anticoagulants in obese patients: guidance from the SSC of the ISTH," Journal of Thrombosis and Haemostasis, vol. 14, no. 6, pp. 1308-1313, 2016.

[63] M. Heisen, M. J. Treur, H. E. Heemstra, E. B. W. Giesen, and M. J. Postma, "Cost-effectiveness analysis of rivaroxaban for treatment and secondary prevention of venous thromboembolism in the Netherlands," Journal of Medical Economics, vol. 20, no. 8, pp. 813-824, 2017.

[64] M. S. Marcolino, C. A. Polanczyk, A. C. C. Bovendorp et al., "Economic evaluation of the new oral anticoagulants for the prevention of thromboembolic events: a cost-minimization analysis," São Paulo Medical Journal, vol. 134, no. 4, pp. 322-329, 2016.

[65] M. S. Jacobs, L. A. de Jong, M. J. Postma, R. G. Tieleman, and M. van Hulst, "Health economic evaluation of rivaroxaban in elective cardioversion of atrial fibrillation," The European Journal of Health Economics, vol. 19, no. 7, pp. 957-965, 2018.

[66] G. Gourzoulidis, G. Kourlaba, J. Kakisis et al., "Cost-effectiveness analysis of rivaroxaban for treatment of deep vein throm- bosis and pulmonary embolism in Greece," Clinical Drug Investigation, vol. 37, no. 9, pp. 833-844, 2017.

[67] K. P. Fung, K. H. Chan, V. Ng, P. T. Tsui, and J. H. S. You, "Health economic analysis of rivaroxaban and warfarin for venous thromboembolism management in Chinese patients," Cardiovascular Drugs and Therapy, vol. 33, no. 3, pp. 331-337, 2019.

[68] J. Salcedo, J. W. Hay, and J. Lam, "Cost-effectiveness of rivaroxaban versus warfarin for treatment of nonvalvular atrial fibrillation in patients with worsening renal function," International Journal of Cardiology, vol. 282, pp. 53-58, 2019.

[69] A. Li, P. M. Manohar, D. A. Garcia, G. H. Lyman, and L. M. Steuten, "Cost effectiveness analysis of direct oral anticoagulant (DOAC) versus dalteparin for the treatment of cancer associated thrombosis (CAT) in the United States," Thrombosis Research, vol. 180, pp. 37-42, 2019.

[70] Z. Ademi, E. Zomer, A. Tonkin, and D. Liew, "Cost-effectiveness of rivaroxaban and aspirin compared to aspirin alone in patients with stable cardiovascular disease: an Australian perspective," International Journal of Cardiology, vol. 270, pp. 54-59, 2018.

[71] A. Duran, N. Sengupta, A. Diamantopoulos, F. Forster, L. Kwong, and M. Lees, "Cost effectiveness of rivaroxaban versus enoxaparin for prevention of post-surgical venous thromboembolism from a U.S. payer's perspective," PharmacoEconomics, vol. 30, no. 2, pp. 87-101, 2012.

[72] E. S. Eerenberg, S. Middeldorp, M. Levi, A. W. Lensing, and H. R. Büller, "Clinical impact and course of major bleeding with rivaroxaban and vitamin K antagonists," Journal of Thrombosis and Haemostasis, vol. 13, no. 9, pp. 1590-1596, 2015.

[73] C. S. Miller, A. Dorreen, M. Martel, T. Huynh, and A. N. Barkun, "Risk of gastrointestinal bleeding in patients taking nonvitamin $\mathrm{K}$ antagonist oral anticoagulants: a systematic review and meta-analysis," Clinical Gastroenterology and Hepatology, vol. 15, no. 11, pp. 1674-1683.e3, 2017.

[74] J. Hagii, H. Tomita, N. Metoki et al., "Characteristics of intracerebral hemorrhage during rivaroxaban treatment: comparison with those during warfarin," Stroke, vol. 45, no. 9, pp. 2805-2807, 2014.

[75] M. Turner and D. Solarz, "Left atrial thrombus formation after brief interruption of rivaroxaban," The American Journal of Emergency Medicine, vol. 34, no. 1, p. 116.e3-4, 2016.

[76] W. Gogarten, K. Hoffmann, and H. Van Aken, "Recommendations for the administration of conventional and new antithrombotic agents from the perspective of anesthesiology," Unfallchirurg, vol. 113, no. 11, pp. 908-914, 2010.

[77] W. Mueck, D. Kubitza, and M. Becka, "Co-administration of rivaroxaban with drugs that share its elimination pathways: pharmacokinetic effects in healthy subjects," British Journal of Clinical Pharmacology, vol. 76, no. 3, pp. 455-466, 2013.

[78] E. C. Christopoulou, T. D. Filippatos, and M. S. Elisaf, "Nonhemorrhage-related adverse effects of rivaroxaban," Archives of Medical Science - Atherosclerotic Diseases, vol. 2, pp. e108e112, 2017.

[79] S. J. Connolly, M. Crowther, J. W. Eikelboom et al., "Full study report of andexanet alfa for bleeding associated with factor Xa inhibitors," The New England Journal of Medicine, vol. 380, no. 14, pp. 1326-1335, 2019.

[80] A. Cuker, A. Burnett, D. Triller et al., "Reversal of direct oral anticoagulants: guidance from the anticoagulation forum," American Journal of Hematology, vol. 94, no. 6, pp. 697-709, 2019. 